\title{
Solution of the First-Passage Problem by Advanced Monte Carlo Simulation Technique
}

\author{
M. Labou \\ Institute of Structural Mechanics, Kiev State University of Construction and Architecture, \\ Kiev, Ukraine \\ удК 539.4

\section{Решение задачи “первого выброса" модифицированным численным методом Монте-Карло}

\section{М. Лабу}

Институт строительной механики при Киевском национальном университете строительства и архитектуры, Киев, Украина

Предложен модифицированный численный метод Монте-Карло для оценки надежности нелинейньхх конструкиий, подвергнутых динамическому нагружению. Использование метода позволяет обобиить важные низковероятностные выборки для нелинейных динамических задач. Эффективность данного метода по сравненио с прямом методом Монте-Карло состоит в возможности определения нелинейного стохастического отклика в области очень низких вероятностей.

Ключевые слова: динамическое нагружение, нелинейная динамическая задача, метод Монте-Карло.

Introduction. In a recent review of methods currently available to analyze multidegree-of-freedom systems under stochastic loading, their merits, limitations and potential to be used in engineering practice have been discussed [1]. From this study it becomes clear that only numerical procedures such as Monte Carlo simulation (MCS) techniques (both in their direct and variance reduction form) and the Response Surface Method (RSM) are capable of treating nonlinearities without restriction as well as systems of higher dimension including reliable information on the response distribution tails.

As direct MCS procedures are quite limited with respect to providing accurate information on the response distribution tails, the variance reduction techniques such as importance, directional, adaptive sampling, etc. are used for static structural problems in order to guide the simulation procedures to the region of interest and, hence, to reduce the numerical effort. For dynamic problems, the selection of this region is more involved than for static problems, since the importance measure is generally time variant. Based on a suitable criterion for indicating which realization will most likely lead to failure, the samples in the region of interest may be split, whereas in the region of less interest they may be discarded or may survive. This procedure has been expanded and applied successfully to MDOF systems. 
1. First-Passage Distributions. A general performance measure for structures under stochastic dynamic loading is characterized by first-passage probabilities, where the distribution of the exit times $T$ into a state of failure is considered. The boundary separating the safe state from the unsafe one might be specified by a so-called limit state function $g(x)=0$. The lifetime of the structure is then defined as the first exit time $T$ :

$$
T=\inf [t: g(X(t)) \leq 0, t \geq 0]
$$

and the probability of the first excursion $P_{f}(t)$ within the time interval $t$ is defined as

$$
P_{f}(t)=P[T \leq t]
$$

specifying the evolution of the failure probability with respect to time.

Presently, the available solutions to the above first probability are quite rare. There is a complete absence of analytical exact solutions in closed form, and numerical procedures based on the Fokker-Planck equation are confined to problems of low dimension $(<4$ in the state space). The only solution technique at hand seems to be the MCS, which is well suited to simulate nonlinear stochastic structural responses as required in the reliability assessment of structures.

2. Method of Analysis. For the purpose of assessing the reliability of a structure, the domain of the response is divided into the safe and unsafe parts, respectively. The so-called limit state function (LSF) serves as a criterion of separation. The domain is in general multidimensional containing parameters such as displacements, velocities, plastic deformations, etc. If $x$ is defined as the state vector of all the response components and $g(x)$ as the LSF, it is necessary to determine the probability $P(t)$ that the response vector $x(t)$ exceeds the LSF at least once within a given time period $[0, t]$, where $x(0)$ lies initially in the safe domain, i.e., $P(0)=0$. In other words, the first-passage probability defines the evolution of the failure probability with respect to time.

It is well known, that the direct MCS is well suited to obtain, with a relatively small number of realizations, reasonably close estimates for the mean vector and the covariance matrix. It is not suited, however, to assess the low probability domain of the stochastic response and for reliability analysis where failure probabilities $P_{f}$ are of the order $10^{-8}-10^{-4}$. In order to access such ranges, it is obvious that a sample size (number of realizations) of the order $10^{5}-10^{9}$ will be required. Such sample sizes are possible for small systems using a massively-parallel supercomputer [2]. However, the MCS with a sample size of the order $>10^{5}$ are expensive and certainly not efficient in view of the fact that only a small fraction of the number of realizations falls into the domain of interest for the reliability analysis.

This drawback of the direct MCS has been commonly recognized, and the so-called variance reduction techniques have been developed and utilized successfully. All these procedures increase the density of realizations in the region of interest, i.e., in the region that contributes most to the failure 
probability. These variance reduction methods are used mainly for nonlinear static reliability systems, but can be also used for nonlinear dynamic problems. The main difficulty with these approaches is the fast growing numerical effort with the number of random variables involved. In case loading is represented by a stochastic process, it might be possible in some cases to represent the stochastic process by a small number of random variables using the Karhunen Loeve expansion.

Monte Carlo simulation, however, is applicable to stochastic loading involving more than just a small set of random variables. In such cases, the traditional variance reduction procedures are no longer applicable.

Advanced MCS methods have been developed for the class of systems with Markovian properties. Before describing the details and implementation of such procedures, we shall briefly discuss the main features of the advanced MCS. Consider first the MCS and the resulting approximation for the cumulative distribution function $C D F(x)$ :

$$
C D F(x, t)=\sum_{n=1}^{N} I\left[X_{n}(t), x\right] w_{n}(t),
$$

where $x$ is the state vector, $t$ is time, $X_{n}$ is the state vector of the $n$th realization, $w_{n}(t)$ is the weight and discrete probability of the $n$th realization at time $t$, and $N$ is the sample size. The indicator function $I\left[X_{n}(t), x\right]$ takes the value 1 if the components $X_{n, k}>x_{k}, k=1, \ldots, M$, for all components $M$ of the state vector. Otherwise, the indicator function assumes the value 0 . In case the direct MCS is applied, all weights are constant and assume the value $w_{n}(t)=$ $=1 / N$. For example, for importance sampling, the original probability density function $f(x)$, which reflects the direct MCS, is modified by using the sampling distribution $h(x)$. This can be done without violating the original density function $f(x)$ by modifying the weight $w_{n}$ of all realizations $X_{n}(t)$ by $w_{n}=$ $=f(x) /(N h(x))$. Since the advanced MCS is designed to assess low probability ranges of the order $10^{-8} \leq P_{f} \leq 10^{-4}$ as well, it is obvious that manipulation of the weights is indispensable.

A selective Monte Carlo simulation technique, namely, "Russian Roulette \& Splitting" simulation technique (RR\&S) has been applied successfully in nuclear physics [3] to solve neutron transport problems. The basic features of the algorithm are described and discussed.

The RR\&S procedure involves subdivision of the safe region by several borders of splitting (or sub-barriers). Then the process of splitting can be introduced as follows. When the response sample function $x(t)$ upcrosses the $i$ th sub-barrier, the state vector is split into $m_{i}$ same vectors, where $m$ is the integer number and $i$ is the number of the sub-barrier. The weight of each new vector is equal to $w_{\text {new }}=w_{\text {old }} / m_{i}$. Due to the mi stochastically independent sample functions produced by splitting, one simply increases the chances to upcross the next (more highly placed) sub-barrier. Thus the splitting technique makes it possible to increase significantly the number of samples which are capable of crossing the limit state function. 
The Russian Roulette is a well-known game of chance where the 'probability of death' is high (i.e., $1 / 6$, originally). This game can be played to reduce the sample size to a required amount. The "Russian Roulette" technique is applied in an inverse situation, i.e., when the response sample function downcrosses the th sub-barrier. In this case with a probability of $1-1 / m_{i}$, the simulation process is discarded (killed) and with a probability of $1 / m_{i}$ the simulation is continued. The weight of such surviving sample is multiplied by $m_{i}$. Hence, the main aim of the "Russian Roulette" technique is to diminish drastically the number of sample functions which are of "low interest" in the first passage problem.

From the algorithm described above it becomes obvious that between the ith and $(i+1)$ th sub-barriers the weights of the samples are equal to each other and are expressed as

$$
w_{i}=\frac{1}{n} \prod_{j=1}^{i} \frac{1}{m_{j}},
$$

where $n$ is the number of initially simulated samples and $m_{j}$ is the number of the splitting on the $j$ th sub-barrier.

It is clear that the weights of the samples $w_{b}$ after outcrossing the barrier will be equal to

$$
w_{b}=\frac{1}{n} \prod_{j=1}^{k} \frac{1}{m_{j}},
$$

where $k$ is the total number of sub-barriers.

It is noteworthy that the direct Monte Carlo algorithm is a subset of the RR\&S technique. In particular, the direct MCS can be obtained from the RR\&S for $m_{i}=1$ [4].

3. Determination of the Effectiveness of the Simulation Technique. According to Rubinstein [5], the effectiveness $E$ of each simulation technique can be expressed in the following form:

$$
E=\frac{1}{\operatorname{var}\left[P^{*}\right] T},
$$

where $\operatorname{var}\left[P^{*}\right]$ is the variance of the estimate and $T$ is the computation time of the estimate $P^{*}$.

The Bernoulli scheme of independent trials in the case of straightforward MCS allows one to obtain the following well-known formula for the variance of the estimate:

$$
\operatorname{var}\left[P^{*}\right]=\frac{P(1-P)}{N}
$$

where $N$ is the sample size. 
Unfortunately, due to the correlation between samples in the RR\&S technique, there is a difficulty in obtaining an expression for the estimate variance similar to (7).

Based on the sample statistics, the variance of estimate for the RR\&S technique can be calculated by the following formula:

$$
\operatorname{var}\left[P^{*}\right]=\frac{1}{n(n-1)} \sum_{i=1}^{n}\left(\frac{l_{i}}{M}-P^{*}\right)^{2},
$$

where

$$
\begin{gathered}
P^{*}=\frac{1}{n} \sum_{i=1}^{n} \frac{l_{i}}{M}, \\
M=\prod_{j=1}^{k} m_{j},
\end{gathered}
$$

$n$ is the sample size, $l_{i}$ is the number of barrier outcrossings in the ith independent trial, and $m_{j}$ is the number of the splitting on the $j$ th sub-barrier.

As long as variance (7) has already been minimized for a given sample size $N$ by the straightforward Monte Carlo simulation, a more effective solution can be obtained using another simulation scheme (8) with a higher variance of the estimate and much shorter computation time.

4. Numerical Examples. Nonlinear vibrations of a cylindrical panel under the action of a uniformly distributed load are analyzed; the time history is described by a truncated white noise. It is considered that at the initial instant of time the cylindrical panel is in a quiescent mode. The action of the applied load triggers the excitation of a nonstationary stochastic process.

The following parameters of the panel are adopted: the dimensions in plan $a=0.2 \mathrm{~m}, b=0.3 \mathrm{~m}$, the height $h=0.002 \mathrm{~m}$, the modulus of elasticity $E=70.63 \mathrm{GPa}$, the density of the material $\rho=2.7 \cdot 10^{3} \mathrm{~kg} / \mathrm{m}^{3}$, Poisson's ratio $v=1 / 3$, and radius of curvature $R=0.8363 \mathrm{~m}$.

In the problem under study, the frequency band of the spectral density of the loading covers the first, fifth, seventh, and eleventh symmetrical vibration modes.

The equation of motion is governed by the following differential equation:

$$
\begin{gathered}
\ddot{x}_{i}+C_{i} \dot{x}_{i}+K_{i} x_{i}+K_{2}^{i j k} x_{j} x_{k}+K_{3}^{i j k l} x_{j} x_{k} x_{l}=\mu_{i} Q_{i}(t) \\
(i, j, k, l=1, \ldots, 4),
\end{gathered}
$$

where $C_{i}$ are factors of the damping matrix, $\mu_{i}$ are the oscillation modes, $Q(t)$ is the Gaussian white noise with intensity $I=1000 \mathrm{~m}^{2} / \mathrm{s}^{3}$, and $K^{i j k}$ and $K^{i j k l}$ $(i, j, k, l=1, \ldots, 4)$ are, respectively, the coefficients of matrices of square and cubic nonlinearity. 
The following input data are considered: $K_{1}=\varpi_{1}^{2}=1.00, K_{2}=\varpi_{2}^{2}=7.64$, $K_{3}=\varpi_{3}^{2}=11.2, \quad K_{4}=\varpi_{4}^{2}=22.00, C_{1}=0.0159, C_{2}=0.044, C_{3}=0.0532$, $C_{4}=0.0747, \mu_{1}=-0.0297, \mu_{2}=0.0095, \mu_{3}=0.00979, \mu_{4}=0.00342$. formula

The flexures in the center of the cylindrical panel are determined by the

$$
x_{c}(t)=h \sum_{i=1}^{4} x_{i}(t) .
$$

The first passage probabilities of the system response $x_{c}(t)$ were determined during time $[0, t]$ for double-sided symmetrical barriers located at the levels $\pm 3 h, \pm 4 h$, and $\pm 5 h$ and shown in Fig. 1 .
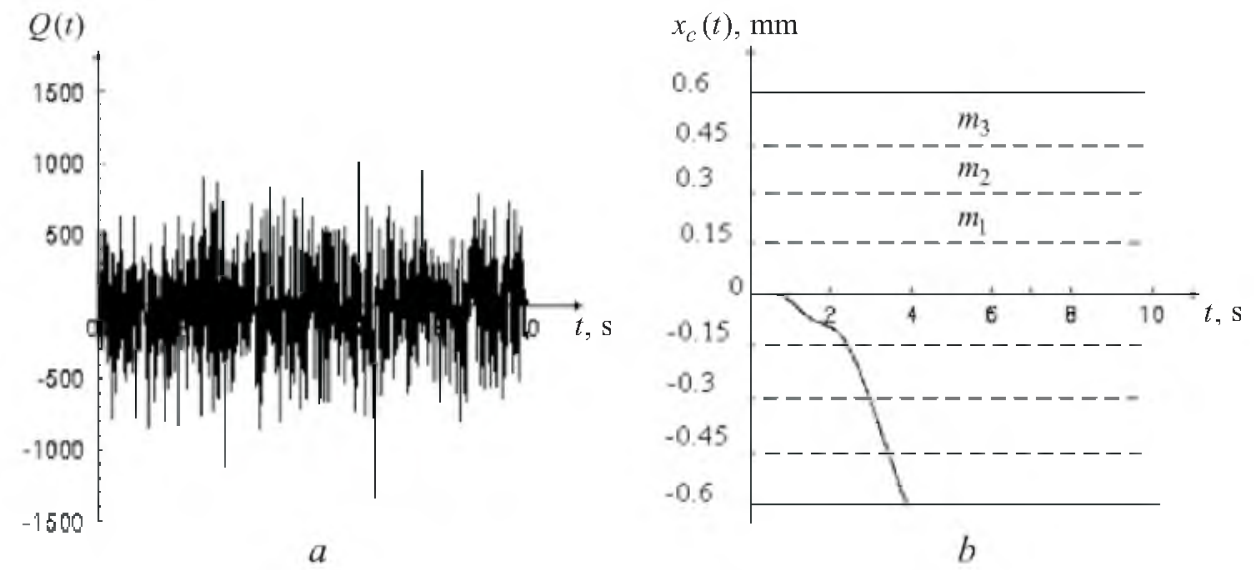

Fig. 1. Sample white noise function $Q(t)-a$ and the system response variation $x_{c}(t)-b$.

At the beginning, the first passage probability for symmetrical barriers of level $\pm 3 h$ was determined.

Figure 1a shows one realization of the stochastic process $Q(t)$. The corresponding variation of the system response $x_{c}(t)$ is presented in Fig. $1 \mathrm{~b}$. This trajectory reaches the limiting barrier at $t=4 \mathrm{~s}$. In the same figure, the arrangement of borders of splitting and the numbers of the splitting are shown. In this case, 6 borders of splitting were located at $\pm 0.15 \mathrm{~cm}, \pm 0.3 \mathrm{~cm}$, and $\pm 0.45 \mathrm{~cm}$; the number of the splitting at each of the borders are $m_{1}=m_{2}=m_{3}=7$; the sample size $n=1000$.

Figure 2 (curve $a$ ) presents in a logarithmic scale the cumulative distribution function of the first passage probabilities of the system response $x_{c}(t)$ for barriers located at the level $\pm 3 h$. From this figure one can see that at $t<4 \mathrm{~s}$ the probability of reaching the barrier sharply decreases. The appropriate events are rather rare

We also analyzed the problems on the first passage probability of the system response $x_{c}(t)$ for barriers located at the levels $\pm 4 h$ and $\pm 5 h$. The appropriate cumulative distribution functions are shown in Fig. 2 (curves $b$ and $c$, respectively). 
$\mathrm{T}$ a b 1 e 1

The Effectiveness and Calculation Time Ratios - MCS to RR\&S

\begin{tabular}{|c|c|c|}
\hline Time (s) & $E_{R R \& S} / E_{M C S}$ & $T_{M C S} / T_{R R \& S}$ \\
\hline 2.22 & 169.00 & 169.0 \\
\hline 2.50 & 4.20 & 149.0 \\
\hline 3.00 & 1.10 & 83.0 \\
\hline 4.00 & 0.40 & 38.0 \\
\hline 5.00 & 0.17 & 22.4 \\
\hline
\end{tabular}

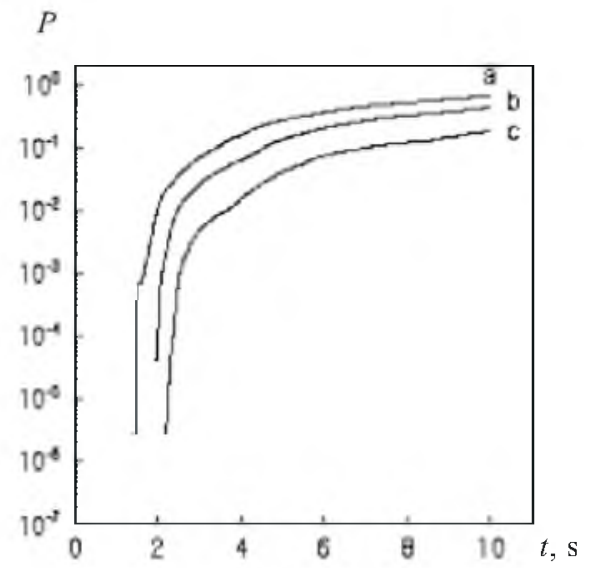

Fig. 2

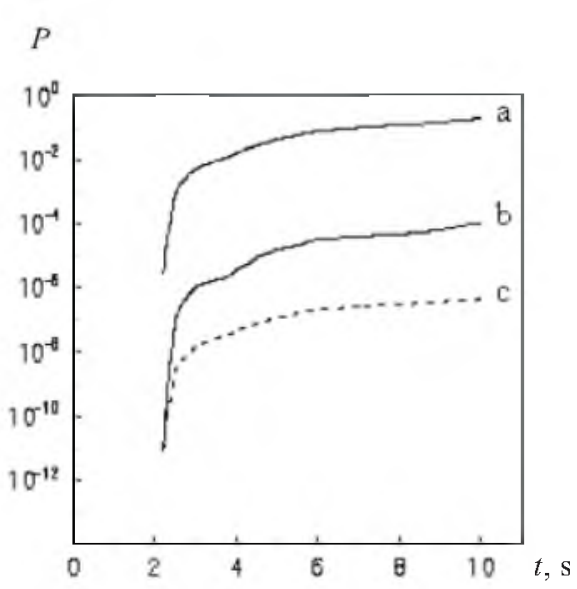

Fig. 3

Fig. 2. Estimates of the first passage probabilities versus time for the double-sided barriers located at $a= \pm 3 h, b= \pm h, c= \pm 5 h$.

Fig. 3. Estimates of the first-passage probabilities and variances of estimates for barriers located at $\pm 5 h$

In the second problem (barriers at the level $\pm 4 h$ ) 6 borders of splitting were located at distances $\pm 0.2 \mathrm{~cm}, \pm 0.4 \mathrm{~cm}$, and $\pm 0.6 \mathrm{~cm}$, the numbers of the splitting at each of the borders are $m_{1}=m_{2}=m_{3}=7$; the cumulative distribution function for this problem is shown in Fig. 2 (curve $b$ ).

In the third problem (barriers at the level $\pm 5 h$ ) 6 borders of splitting were located at distances $\pm 0.25 \mathrm{~cm}, \pm 0.5 \mathrm{~cm}$, and $\pm 0.75 \mathrm{~cm}$, the number of the splitting at each of the borders are $m_{1}=m_{2}=m_{3}=7$, the appropriate cumulative distribution function is shown in Fig. 2 (curve $c$ ). The sample size $n$ in the second and third problem equals to 1000 .

For the barriers located at $\pm 5 h$, the variance of estimates $P^{*}$ was calculated for different techniques. Figure 3 (curve $a$ ) represents cumulative distribution functions obtained successively by the straightforward MCS (dotted lines), and the RR\&S procedure (solid lines) (see Fig. 2, curve c).

Figure 3 (curve $b$ ) represents the variance of estimates $P^{*}$, obtained with the help of formula (8); and curve (c) obtained using formula (7). The ratio of the effectiveness $E_{R R \& S} / E_{M C S}$ calculated for different times $t$ in accordance with (6) is presented in Table 1 together with the ratio of the calculation times $T_{M C S} / T_{R R \& S}$. 
From Table 1 it is seen that the RR\&S simulation technique offers a considerable gain in computation time and a higher effectiveness (in the first $3 \mathrm{~s}$ ) as compared to the direct MCS technique.

Conclusion. An advanced Monte Carlo simulation procedure has been applied to MDOF systems showing its basic applicability to higher dimensional problems and the ability to assess the low probability range in terms of the first-passage probabilities.

\section{Резюме}

Запропоновано модифікований числовий метод Монте-Карло для оцінки надійності нелінійних конструкцій під дією динамічного навантаження. Метод дозволяє узагальнити важливі низькоімовірнісні вибірки для нелінійних динамічних задач. Ефективність даного методу в порівнянні 3 прямим методом Монте-Карло полягає в можливості визначення нелінійного стохастичного відгуку в області дуже низьких імовірностей.

1. G. I. Schuller, H. J. Pradlwarter, and M. D. Pandey, "Methods for reliability of nonlinear systems under stochastic loading - a review," Proc. EURODYN'93, Balkema (1993) pp. 751-759.

2. E. A Johnson E. A. and L. A. Bergmann, "Monte Carlo simulation of dynamic systems of engineering interest in a massively parallel computing environment," in: Proc. IUTAM Symp. "Advances in Nonlinear Stochastic Mechanics," (Trondheim, Norway, July 3-7, 1995), Kluwer Academic Publishers, Dordrecht, the Netherlands (1996), pp. 225-234.

3. J. Spanier and E. M. Gelbard, Monte Carlo Principles and Neutron Transport Problems, Addison Wesley Publishing Company (1969).

4. P. G. Mel'nik-Mel'nikov, E. S. Dekhtyaruk, and M. Labou, “Application of the "Russian Roulette and Splitting" simulation technique for the reliability assessment of mechanical systems," Probl. Prochn., No. 3, 131-135 (1997).

5. R. Y. Rubinstein, Simulation and the Monte Carlo Method, Wiley, New York (1981). 